\title{
A Complicated Ileal Duplication Cyst in a Young Adult: The Value of the "Gut Signature"
}

\author{
Marco Di Serafino ${ }^{*}$, Rosa Severino ${ }^{2}$, Carmela Mercogliano ${ }^{3}$, Francesco Lisanti ${ }^{4}$, \\ Ciro De Martino ${ }^{5}$, Rosario Rocca ${ }^{1}$, Rosaria Abate ${ }^{3}$, Michele Salata ${ }^{3}$, Gianfranco Vallone ${ }^{6}$, \\ Domenico Maroscia ${ }^{1}$ \\ ${ }^{1}$ Emergency Radiology Department, San Carlo Hospital, Potenza, Italy \\ ${ }^{2}$ Radiology Department, Federico II University Hospital, Naples, Italy \\ ${ }^{3}$ Women and Child Department, San Carlo Hospital, Potenza, Italy \\ ${ }^{4}$ Emergency-Urgency Department, San Carlo Hospital, Potenza, Italy \\ ${ }^{5}$ Emergency Surgery Department, San Carlo Hospital, Potenza, Italy \\ ${ }^{6}$ Pediatric Radiology Department, Federico II University Hospital, Naples, Italy \\ Email: *marcodiserafino@hotmail.it
}

Received 2 May 2016; accepted 3 June 2016; published 6 June 2016

Copyright (C) 2016 by authors and Scientific Research Publishing Inc.

This work is licensed under the Creative Commons Attribution International License (CC BY).

http://creativecommons.org/licenses/by/4.0/

(c) (i) Open Access

\begin{abstract}
Intestinal duplication is an uncommon congenital abnormality, with potential life-threatening complications, usually manifesting in childhood. Due to its non-specific clinical presentation as well as its resemblance to other abdominal processes at imaging, the differential diagnosis may be very difficult. This case report describes a young female adult accessed twice to our Emergency Department with recurrent pain in the right iliac fossa and a clinical picture of abdominal pain and bowel obstruction, who was finally diagnosed with complicated duplication cyst, then resected. The diagnostic tools performed show the simple duplication cyst at the beginning, in the ileocecal region, with the characteristic "gut signature" at Ultrasound and its subsequent inflammatory changing at Computed Tomography, which led to the right diagnostic hypothesis, confirmed by histopathology. An early diagnosis of this condition is needed to undertake the correct surgical approach.
\end{abstract}

\section{Keywords}

Intestinal Duplication, Gut Signature, Right Iliac Fossa, Abdominal Pain, Complicated Cyst

\footnotetext{
${ }^{*}$ Corresponding author.
}

How to cite this paper: Di Serafino, M., Severino, R., Mercogliano, C., Lisanti, F., De Martino, C., Rocca, R., Abate, R., Salata, M., Vallone, G. and Maroscia, D. (2016) A Complicated lleal Duplication Cyst in a Young Adult: The Value of the "Gut Signature". Open Journal of Radiology, 6, 100-104. http://dx.doi.org/10.4236/ojrad.2016.62015 


\section{Introduction}

Intestinal duplication cyst is a rare congenital abnormality that can occur anywhere in the gastrointestinal tract, from the tongue to the anus, most commonly presenting in the distal ileum; it usually manifests in childhood (80\%), less commonly in adults or young adults. Its presentation is non-specific, with abdominal pain related to abdominal mass or complications, with intussusception being the most frequent [1]. Because of its potential complications an early diagnosis has to be achieved when possible [2] [3]. The main imaging tool is Ultrasound, which can detect a cystic lesion with a quite characteristic appearance of double-layered wall, the so-called "gut signature" [4]. Nevertheless, this sign is not always present, therefore, the lesion may resemble other kind of cystic abnormalities [5]. In this case, a complicated ileal duplication cyst is depicted, first misdiagnosed as mucocele, surgically removed.

\section{Case Report}

A 16 years old girl came to our Emergency Department with recurrent pain in the right iliac fossa and diarrhoea. Accordingly to clinical, biological and endoscopic findings, she was discharged with diagnosis of Crohn disease. During the periodical follow-up for Crohn disease, further instrumental examinations, with Ultrasound (US) and Magnetic Resonance (MR), revealed the presence of an oval lesion measuring about $6 \mathrm{~cm}$ in the right iliac fossa with slightly dense fluid content and enhancing walls (Figure 1 and Figure 2). The hypothesis of mucocele was proposed according to the first diagnosis of chronic inflammatory disease and a "wait and see" approach was then undertaken. Twelve months later, the patient, under an effective treatment for her condition, came again to the Emergency Department with vomit and fever. Laboratory findings revealed high inflammatory markers (PCR: $154 \mathrm{mg} / \mathrm{L}$; WBC: $29.66 \times 10^{3} / \mu \mathrm{L}$ ). The US examination showed a bowel-like structure in the ileocecal area with thickened and hyperaemic walls and loss of stratification. At plain film there was a gas-fluid level in the right iliac fossa (Figure 3). At this stage, clinical and radiological features were suggestive for appendicitis or Crohn recurrence. Therefore, a Computed Tomography scan was performed and it showed a grossly round lesion, measuring about $7 \mathrm{~cm}$, with thick walls and inhomogeneous faeces-like content, located at the level of ileocecal valve (Figure 4). A comparison with previous US and MR was needed and a re-evaluation of images made more clear the presence of a singular sign, known as "gut-signature" on US imaging. It was taken into account the possibility that the lesion, first ascribed to mucocele, was a duplication cyst, subsequently complicated. The patient underwent surgical treatment to remove the lesion (Figure 5). At histological evaluation was confirmed the diagnosis of small bowel duplication cyst, which appeared infected and in continuity with the intestinal lumen. The patient is continuing to do well and is currently undergoing periodic follow-up for Crohn disease as outpatient.

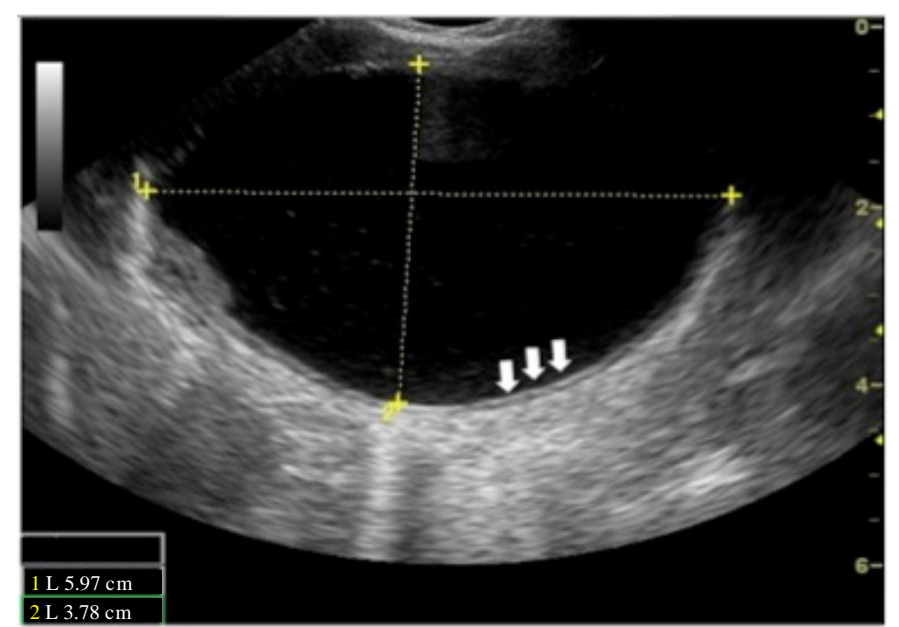

Figure 1. Ultrasound with high linear (10 - 15) $\mathrm{MHz}$ frequency probe and virtual convex setting shows a cystic mass measuring 60 by $38 \mathrm{~mm}$ in diameter. Portions of the cyst wall show the characteristic doublelayered appearance with echogenic mucosa internally and hypoechoic muscle externally (arrows). 


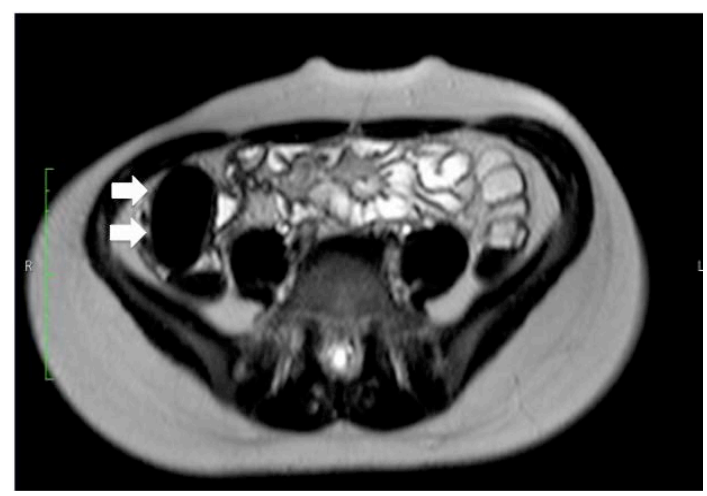

(a)

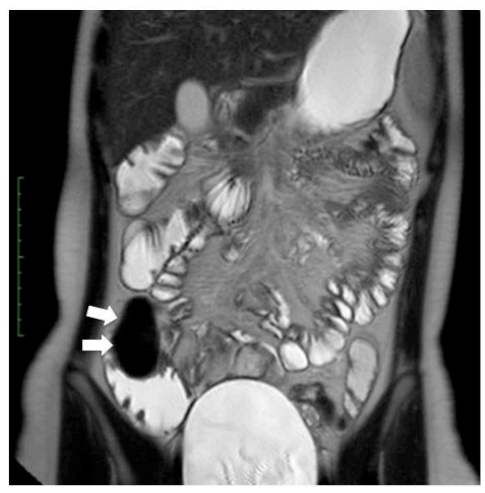

(b)

Figure 2. Entero-MRI, T1w axial (a) and coronal (b) images show oval lesion with hypo-intense signal (arrows), in the ileocecal region, adjacent and not communicating with the bowel.

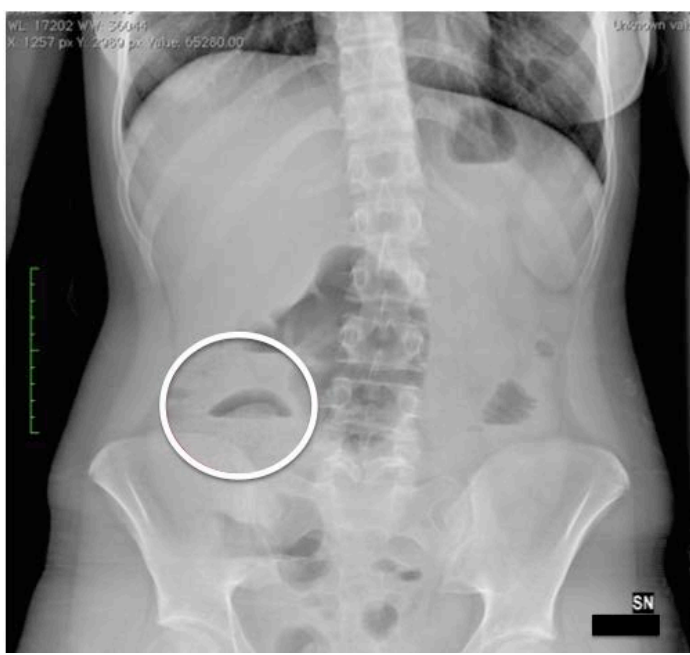

Figure 3. Plain film shows a gas-fluid level in the right iliac fossa (circle).

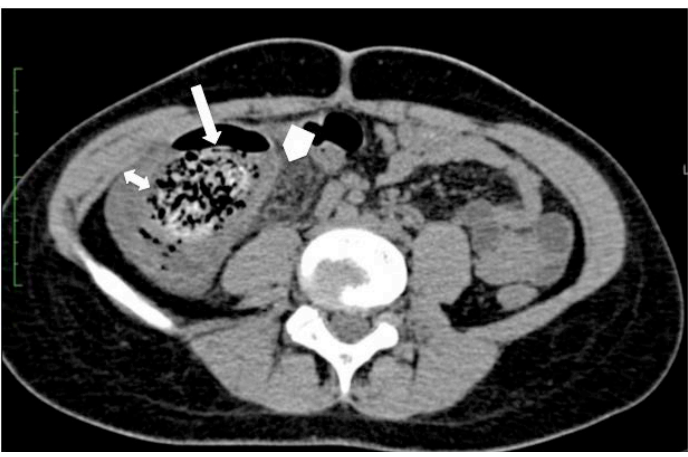

(a)

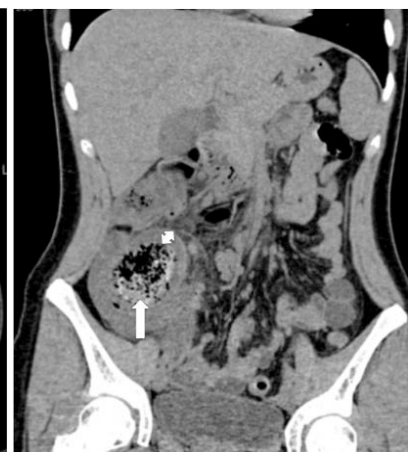

(b)

Figure 4. Non-enhanced CT scan, axial (a) and coronal reconstructions (b) show an oval lesion with thick walls (double head arrows) and mixed gas and faeces-like content (arrows), surrounded by reactive fat thickening (arrowhead).

\section{Discussion}

Intestinal duplications are uncommon congenital anomalies that can occur anywhere in the gastrointestinal tract. The most common site is the distal ileus followed by oesophagus, jejunum, stomach and duodenum [1]-[5]. The 


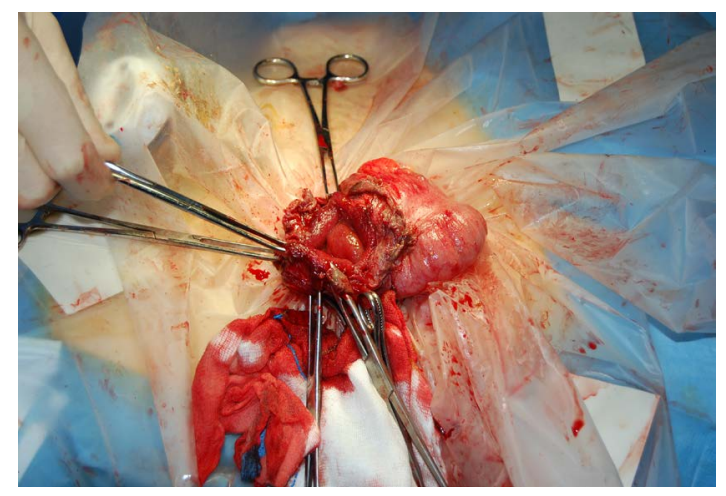

Figure 5. Surgical treatment of the complicated duplication cyst (arrow).

main characteristic feature of the duplication cyst is its wall, which is similar to the intestinal one, with multiple layers including mucosa, sub-mucosa and inner circular muscle layer with associated mesenteric plexus. However, microscopically, the lining mucosa may contain cluster of cells different from the adjacent gastrointestinal tract. Actually, it was found in some cases ectopic gastric or pancreatic tissue [1] [2].

Duplication cysts may appear as spherical or tubular lesions with fluid content, laying close to the mesenteric side of an intestinal tract or strictly connected to the bowel, sharing the muscular layer with its wall [2]-[5].

Although the exact mechanism that leads to the formation of duplication cysts is still uncertain, many authors claim the theory of an abnormal recanalization of the gut lumen. It seems that, during embryogenesis, after an intense epithelial proliferation inside the gut, the formation and then the coalescence of vacuole-like structures makes the bowel patent again. According to this hypothesis, an incomplete merging of vacuoles, during the recanalization of the gut tube, may be responsible for duplication cyst formation [2]-[5].

Classic presentation of these congenital anomalies is both as spherical or tubular lesions. The former, more common (80\%), typically do not communicate with the intestinal lumen, whereas the tubular variant, infrequent (20\%), is in direct connection with the bowel lumen [2]-[5].

Even though there are not specific symptoms, most duplication cysts are detected during the first year of life. They can early manifest because of the development of potential life-threatening conditions such as obstruction or intussusception. In other cases they cause palpable mass, abdominal pain or, in presence of ectopic gastric tissue, may be responsible for peptic ulcers and haemorrhage. Further described complications are infection and perforation [2]-[6].

Radiologically, plain film can show aspecific signs of bowel obstruction or intussusception when complicated [2]. Ultrasound plays a critical role in the detection of intestinal duplication cysts. In fact, it can image a characteristic appearance of the duplication cyst, which is a double-layered wall, also known as "gut signature", with inner hyperechoic mucosa and outer hypoechoic muscle layer [1]. However, the "gut signature" is not a specific sign of duplication cyst as it could be also found in cysts of other nature, such as ovarian or mesenteric cysts and Meckel's diverticula [4] [5]. In addition, in some cases this sign could be not detectable (50\%) [1]-[5]. Despite these considerations, recognising a bi-layer walled cyst, especially when located adjacent to the bowel, is highly suggestive of intestinal duplication [1]-[4].

In our case, at last US study it was possible to identify a cystic lesion adjacent to the small bowel with vascularised wall at Colour-Doppler analysis, while it was not detectable a double-layer appearance probably because of intense inflammatory changing [4]. Cheng et al. (2005) argued that a false negative "gut signature" might be related to the erosion of the internal hyperechoic layer and thickening of the hypoechoic one, corresponding to exudate and inflamed connective tissue into a complicated cyst [7].

CT scan is usually not performed for the evaluation of duplication cysts, however, it could be necessary in case of complications or to define the cyst's extension [3]. At CT small bowel duplication cysts appear as fluidfilled cystic lesion with thick and enhancing wall, strictly adjacent to the ileum. The internal attenuation values are both described to be fluid-like or higher, probably when proteinaceous and/or haemorrhagic component are present in cysts complicated by infection [5] [6]. This was the case of the young patient we described, who also manifested clinical and laboratory signs of inflammatory state. Nevertheless, location of the inflammatory 
changes in the ileocecal area made difficult the differential diagnosis from appendicitis, as it was not easy to detect the normal appendix at both US and CT. Other differential diagnoses to consider were complications of Crohn disease and Meckel's Diverticulum. This latter is usually excluded because of its typical location in the anti-mesenteric side of the ileum, whereas duplication cysts may occur in the opposite margin. However, are described in the literature several cases of this kind of misdiagnosis [1]. Moreover, in our case, at first evaluation of the young patient it was not recognised the "gut signature" at US, and the presence of a cystic lesion near the ileocecal valve was misinterpreted as mucocele according to her underlying Crohn disease.

The suggested treatment for duplication cyst is surgery, mainly to avoid possible complications but also to confirm the diagnosis [5] [6].

After surgical excision, histopathology can verify the nature of the cyst.

\section{Conclusion}

In conclusion, this report describes a rare case of duplication cyst, a potentially life-threatening condition, uncommonly manifesting in a young adult patient. Despite the diagnosis can be strongly suggested by the presence of a quite typical "gut signature" [6], it was missed at first evaluation with US and MR, as the appearance of this congenital lesion may resemble cyst of other nature, especially if located close to the ileocecal region. Only at a second access to the Emergency Department, the lesion was retrospectively ascribed to duplication cyst subsequently complicated. A correct diagnosis is important for these patients, because of the risk of complications and the necessity of surgical resection, which is thought to be the right therapeutic approach [1].

\section{Acknowledgements}

The Authors declare that no conflict of interest exists with the results and conclusions presented in this paper. Publication ethics have been observed.

\section{References}

[1] Puligandla, P.S., Nguyen, L.T., St-Vil, D., Flageole, H., Bensoussan, A.L., Nguyen, V.H. and Laberge, J.M. (2003) Gastrointestinal Duplications. Journal of Pediatric Surgery, 38, 740-744. http://dx.doi.org/10.1016/jpsu.2003.50197

[2] Macpherson, R.I. (1993) Gastrointestinal Tract Duplications: Clinical, Pathologic, Etiologic, and Radiologic Considerations. Radiographics, 13, 1063-1080. http://dx.doi.org/10.1148/radiographics.13.5.8210590

[3] Liu, R. and Adler, D.G. (2014) Duplication Cysts: Diagnosis, Management, and the Role of Endoscopic Ultrasound. Endosc Ultrasound, 3, 152-60. http://dx.doi.org/10.4103/2303-9027.138783

[4] Di Serafino, M., Mercogliano, C. and Vallone, G. (2016) Ultrasound Evaluation of the Enteric Duplication Cyst: The Gut Signature. Journal of Ultrasound, 19, 131-133. http://dx.doi.org/10.1007/s40477-015-0188-8

[5] Lee, N.K., Kim, S., Jeon, T.Y., Kim, H.S., Kim, D.H., Seo, H.I., Park do Y. and Jang, H.J. (2010) Complications of Congenital and Developmental Abnormalities of the Gastrointestinal Tract in Adolescents and Adults: Evaluation with Multimodality Imaging. Radiographics, 30, 1489-1507. http://dx.doi.org/10.1148/rg.306105504

[6] Tong, S.C., Pitman, M. and Anupindi, S.A. (2002) Best Cases from the AFIP. Ileocecal Enteric Duplication Cyst: Radiologic-Pathologic Correlation. Radiographics, 22, 1217-1222. http://dx.doi.org/10.1148/radiographics.22.5.g02se221217

[7] Cheng, G., Soboleski, D., Daneman, A., Poenaru, D. and Hurlbut, D. (2005) Sonographic Pitfalls in the Diagnosis of Enteric Duplication Cysts. AJR, 184, 521-525. http://dx.doi.org/10.2214/ajr.184.2.01840521 